\title{
Is nutrient intake associated with physical activity levels in healthy young adults?
}

Yi Yan ${ }^{1, *}$, Clemens Drenowatz ${ }^{2}$, Gregory A Hand ${ }^{2,3}$, Robin P Shook ${ }^{4}$, Thomas G Hurley ${ }^{5}$, James R Hebert ${ }^{5,6}$ and Steven N Blair 2,6

'Sports Science College, Beijing Sport University, No. 48 Xinxi Road, Haidian District, Beijing 100084, People's Republic of China: ${ }^{2}$ Department of Exercise Science, Arnold School of Public Health, University of South Carolina, Columbia, SC, USA: ${ }^{3}$ School of Public Health, West Virginia University, Morgantown, WV, USA: ${ }^{4}$ Department of Kinesiology, College of Human Sciences, lowa State University, Ames, IA, USA: ${ }^{5}$ South Carolina Statewide Cancer Prevention and Control Program, Arnold School of Public Health, University of South Carolina, Columbia, SC, USA: ${ }^{6}$ Department of Epidemiology and Biostatistics, University of South Carolina, Columbia, SC, USA

Submitted 5 June 2015: Final revision received 14 November 2015: Accepted 14 December 2015: First published online 22 February 2016

\begin{abstract}
Objective: Both physical activity (PA) and diet are important contributors to health and well-being; however, there is limited information on the association of these behaviours and whether observed associations differ by weight. The present study aimed to evaluate whether nutrient intake is associated with PA and if this association varies by weight in young adults.

Design: Cross-sectional study to analyse the association between PA and nutrient intake.

Setting: Participants were stratified as normal weight $\left(18.5 \mathrm{~kg} / \mathrm{m}^{2}<\right.$ BMI $\left.<25.0 \mathrm{~kg} / \mathrm{m}^{2}\right)$ and overweight/obese $\left(\mathrm{BMI} \geq 25 \cdot 0 \mathrm{~kg} / \mathrm{m}^{2}\right)$. PA level (PAL) was calculated $(\mathrm{PAL}=$ total daily energy expenditure/RMR) and used to stratify groups (PAL $<1.6,1.6 \leq \mathrm{PAL}<1.9$, PAL $\geq 1.9$ ).

Subjects: Adults ( $n$ 407; age 27.6 (sD 3.8) years, $48 \%$ male), with BMI between 20 and $35 \mathrm{~kg} / \mathrm{m}^{2}$, having at least two $24 \mathrm{~h}$ diet recalls and at least $5 \mathrm{~d}$ (including two weekend days) of valid, objectively measured PA data were included in the analysis.

Results: In normal-weight participants, higher PAL was associated with higher intakes of minerals (except $\mathrm{Ca}, \mathrm{Fe}$ and $\mathrm{Zn}$ ), B-vitamins and choline ( $P$ for trend $<0 \cdot 05)$. In the overweight/obese group, higher PAL was associated with higher intakes of fibre, $\mathrm{K}, \mathrm{Na}$ and $\mathrm{Cu}(P$ for trend $<0.05)$. These differences, however, were no longer significant after additionally controlling for total energy intake. Conclusions: More active young adults have higher intakes of essential micronutrients. The benefits of PA may be predominantly due to a higher overall food intake while maintaining energy balance rather than a healthier diet.
\end{abstract}

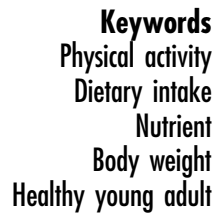

Maintaining a healthy body weight is an effective way to reduce the risk for diabetes, CVD and some cancers ${ }^{(1-3)}$. Poor diet and physical inactivity are key behavioural factors contributing to excess body weight and high body fatness. Adequate nutrient intake is important for optimal health, beyond total energy intake. In fact, it has been argued that dietary patterns such as the Mediterranean diet, with its emphasis on consumption of fruit, vegetables, whole grains and fish, affect body weight independent of energy intake ${ }^{(4)}$. Despite an abundance of foods in the US marketplace, many people do not eat a variety of foods that would provide adequate levels of nutrient intake, as specified in the Dietary Guidelines for Americans, while meeting their energy requirements ${ }^{(5)}$. In addition, energy restriction may further compromise intakes of essential nutrients ${ }^{(6)}$. Of particular concern are low intakes of dietary fibre, $\mathrm{Ca}, \mathrm{K}$ and vitamin $\mathrm{D}$, which are lower than current recommendations in many individuals ${ }^{(5,7)}$, and are associated with obesity ${ }^{(8)}$, hypertension $^{(9,10)}$ and osteoporosis ${ }^{(11)}$.

There is some evidence that physical activity (PA) is associated with a better dietary intake ${ }^{(2,12,13)}$. The benefits of PA regarding nutrient intake, however, have been attributed to an exercise-induced increase in energy requirement that is typically compensated by increased energy intake ${ }^{(14)}$, rather than differences in dietary pattern. Diet information, however, was commonly obtained via diet history questionnaires $^{(2,12)}$, which provide only limited information on current dietary patterns. Most studies further focused on 
the elderly or members of high-risk populations, such as the obese or metabolic syndrome patients ${ }^{(15,16)}$. Currently, there is limited information on the association between nutrient intake and PA in young, healthy adults. The present study examined if nutrient intake varies by PA level (PAL) in adults aged 21-35 years. It was hypothesized that individuals who have higher PAL have higher intakes of key nutrients. Given that PAL and dietary patterns have been shown to differ between normal-weight and overweight/obese individuals $^{(17,18)}$, potential differences in this association by weight status were examined as well.

\section{Methods}

\section{Participants}

The present study used baseline data from an ongoing observational study. The design and rationale for the Energy Balance Study has been described in detail previously $^{(19)}$. Briefly, 430 healthy young adults (48.5\% male; 21-35 years of age) with a BMI between 20 and $35 \mathrm{~kg} / \mathrm{m}^{2}$ were recruited via flyers, email messages on listservs and social media. Inclusion/exclusion criteria were selected to allow for the recruitment of a broad group of individuals with no major acute or chronic diseases. Specifically, exclusion criteria consisted of participants with a resting blood pressure exceeding $150 \mathrm{mmHg}$ systolic and/or $90 \mathrm{mmHg}$ diastolic, an ambulatory blood glucose level greater than $145 \mathrm{mg} / \mathrm{dl}$ or self-reported medication for any major chronic health condition. In addition, participants were excluded if they reported a history of major depression, anxiety disorder or panic disorder. In order to be included in the present analysis participants needed to have had at least two $24 \mathrm{~h}$ diet recalls (24HR) and at least $5 \mathrm{~d}$ (including two weekend days) of valid, objectively measured PA data; resulting in a final sample size of 407 ( $49 \%$ men). The study was approved by the University of South Carolina Institutional Review Board and all participants signed informed consent prior to data collection.

\section{Assessment of body size and composition}

Body weight (in kilograms) and height (in centimetres) were measured according to standard procedures, with participants wearing surgical scrubs and in bare feet. The average of three measurements was used to calculate BMI (BMI = body weight/height ${ }^{2}$ ), which was subsequently used to differentiate between normal-weight $\left(18.5 \mathrm{~kg} / \mathrm{m}^{2}<\mathrm{BMI}\right.$ $<25.0 \mathrm{~kg} / \mathrm{m}^{2}$ ) and overweight/obese individuals (BMI $\geq$ $\left.25.0 \mathrm{~kg} / \mathrm{m}^{2}\right)^{(5,20)}$. Fat mass and lean mass were measured via dual-energy X-ray absorptiometry (Lunar DPX system, version 3.6; Lunar Radiation Corp., Madison, WI, USA).

\section{Assessment of physical activity level}

The SenseWear Mini Armband ${ }^{\circledR}$ (Body Media, Pittsburgh, PA, USA), which has been shown to be a valid device to measure energy expenditure ${ }^{(21,22)}$, was used to measure total daily energy expenditure (TDEE) over a $10 \mathrm{~d}$ period.
Participants were asked to wear the armband for $24 \mathrm{~h}$ except during any periods when the armband could get wet. Participants were deemed compliant if they completed $5 \mathrm{~d}$ of wear (including two weekend days) with at least $19 \mathrm{~h}$ of verifiable time per day. During non-wear time participants were instructed to record their activities, which were matched with the corresponding metabolic equivalent of task (MET) value according to the 2011 Compendium of Physical Activities ${ }^{(23)}$. Energy expenditure missing from the armband was calculated using the participant's RMR.

RMR was measured via indirect calorimetry using a ventilated hood and an open-circuit system (True One 2400; Parvo Medics, Sandy, UT, USA). Participants arrived in the laboratory after a $12 \mathrm{~h}$ fast and at least $24 \mathrm{~h}$ after the last bout of structured exercise. Participants remained awake and rested in a supine position for up to $15 \mathrm{~min}$ prior to measuring expired gases over a $30 \mathrm{~min}$ period.

PAL was calculated (PAL $=$ TDEE/RMR) and used to stratify participants into sedentary or lightly active $(\mathrm{PAL}<1 \cdot 6)$, active or moderately active $(1.6 \leq \mathrm{PAL}<1.9)$ or vigorously active $(\mathrm{PAL} \geq 1 \cdot 9)^{(24)}$.

\section{Nutrient intake and reporting accuracy}

Multiple telephone-administered $24 \mathrm{HR}$ interviews were administered over a period of $14 \mathrm{~d}$, including two weekdays and one weekend day. Prior to the $24 \mathrm{HR}$ participants received portion size training to increase reporting accuracy. The Nutrition Data System for Research software NDSR $^{\circledR}$ version 2012) was used to conduct the dietary interviews. NDSR is considered the state-of-the-art research software for conducting dietary recalls ${ }^{(25)}$. In the present study, 24HR were collected by a team of experienced ( $>6$ years of using NDSR) registered dietitians specifically trained in using the NDSR protocol using the multi-pass approach. The protocol uses prompting to reduce omissions and standardizes the interview method across interviews. Interviews were conducted on randomly selected, non-consecutive days and cold calls were made to participants to minimize preparation that could bias recall. In order to be included in the analysis participants needed to complete at least two diet recalls ${ }^{(26)}$.

In order to adjust for dietary misreporting, the difference between reported energy intake (EI) and energy requirement was used as a covariate (EI difference) in statistical analyses. Energy requirement was calculated based on change in fat mass and fat-free mass over a 3-month period and average $\mathrm{TDEE}^{(27)}$. In addition, scores derived from the MarlowCrowne Social Desirability Scale ${ }^{(28)}$ and the Martin-Larsen Approval Motivation Scale ${ }^{(29)}$ were used to evaluate social desirability and social approval of participants, which have been shown to bias self-reported dietary information ${ }^{(30-33)}$.

\section{Statistical analysis}

Due to potential differences in dietary nutrient intakes and PA, normal-weight and overweight/obese participants 
were analysed separately. Descriptive characteristics were calculated for the three PAL groups within each weight category. The nutrient intake differences between different activity groups were initially examined via MANCOVA, adjusting for sex, age, social desirability, social approval and EI difference; a second analysis adjusted for reported EI, in addition to the previously used covariates. All analyses were carried out using the statistical software package SPSS Version 16 and the nominal significance level for a linear trend across groups was set at $\alpha=0.05$.

\section{Results}

Descriptive characteristics are presented in Table 1. Two-thirds (67\%) of the participants were of European descent, with the majority ( $84 \%$ ) having $\geq 4$ years of college education. In the total sample, $84 \%$ of participants ( $51 \%$ male) were considered active or vigorously active. There were no differences by weight category in race, sex and educational background. However, normal-weight participants were significantly younger than overweight/ obese participants (27.0 (SD 3.6) v. 28.3 (SD 3.8) years; $P<0 \cdot 01)$ and had significantly higher PAL $(P<0 \cdot 01)$. In both normal-weight and overweight/obese groups, more men were vigorously active. There were no differences in anthropometric characteristics across PAL categories in the normal-weight participants while BMI decreased with increasing PAL in overweight/obese ( $P$ for trend $<0 \cdot 01$ ).

Table 2 shows nutrient intakes by activity group, separately for normal-weight and overweight/obese participants. After adjusting for age, sex, social desirability, social approval and EI difference, normal-weight participants had lower intakes of protein, cholesterol,
$\mathrm{P}$, Na, Se, choline $(P<0.01), \mathrm{Ca}$ and $\mathrm{Zn}(P<0.05)$, but higher carbohydrate intake $(P<0.05)$, compared with overweight/obese participants. After additionally controlling for reported EI, normal-weight participants had lower protein $(P<0.05)$ and $\mathrm{Na}$ intakes $(P<0.01)$ but higher intakes of total fibre, $\mathrm{Mg}, \mathrm{Cu}$ and vitamin $\mathrm{E}(P<0 \cdot 01)$ than overweight/obese participants.

After adjusting for age, sex, social desirability, social approval and EI difference, in the normal-weight group, vigorously active participants had significantly higher intakes of $\mathrm{Mg}, \mathrm{P}, \mathrm{Na}, \mathrm{Cu}, \mathrm{Se}$, thiamin, niacin and choline compared with sedentary participants (all $P<0 \cdot 05$; Fig. 1); in overweight/obese participants, except for higher $\mathrm{Na}$ and $\mathrm{Cu}$ intakes $(P<0.05)$, vigorously active participants had similar nutrient intakes to sedentary participants (Fig. 2). Differences were no longer significant after additionally adjusting for reported EI.

\section{Discussion}

Results of the present study suggest that individuals who have higher PAL have higher intakes of key nutrients. After additionally adjusting for reported EI, there was no difference in nutrient intakes across PAL categories, indicating that dietary pattern did not differ by activity level. A higher PAL, however, allows for a higher energy intake while maintaining energy balance.

Various studies have shown a positive association between PAL and concordance with nutrient guidelines ${ }^{(2,34,35)}$ and there is evidence of a positive association between nutrient intake and cardiorespiratory fitness ${ }^{(12,36)}$. The present study also showed higher intakes of key nutrients in active normal-weight participants, but nutrient

Table 1 Descriptive characteristics of the sample of healthy young adult participants from the Energy Balance Study, by activity level in each weight category

\begin{tabular}{|c|c|c|c|c|c|c|c|c|c|c|c|c|}
\hline & \multicolumn{6}{|c|}{ Normal-weight group ( $n$ 210) } & \multicolumn{6}{|c|}{ Overweight/obese group ( $n$ 197) } \\
\hline & \multicolumn{2}{|c|}{$\begin{array}{l}\text { Sedentary } \\
\quad(n 31)\end{array}$} & \multicolumn{2}{|c|}{$\begin{array}{l}\text { Active } \\
(n 102)\end{array}$} & \multicolumn{2}{|c|}{$\begin{array}{l}\text { Vigorously active } \\
(n 77)\end{array}$} & \multicolumn{2}{|c|}{$\begin{array}{l}\text { Sedentary } \\
\quad(n 34)\end{array}$} & \multicolumn{2}{|c|}{$\begin{array}{l}\text { Active } \\
(n 113)\end{array}$} & \multicolumn{2}{|c|}{$\begin{array}{l}\text { Vigorously active } \\
\quad(n 50)\end{array}$} \\
\hline & Mean & SD & Mean & SD & Mean & SD & Mean & SD & Mean & SD & Mean & SD \\
\hline Male (\%) & \multicolumn{2}{|c|}{$29 \cdot 0$} & \multicolumn{2}{|c|}{43.1} & \multicolumn{2}{|c|}{$55 \cdot 8$} & \multicolumn{2}{|c|}{38.2} & \multicolumn{2}{|c|}{$50 \cdot 4$} & \multicolumn{2}{|c|}{$64 \cdot 0$} \\
\hline Female (\%) & \multicolumn{2}{|c|}{71.0} & \multicolumn{2}{|c|}{$56 \cdot 9$} & \multicolumn{2}{|c|}{44.2} & \multicolumn{2}{|c|}{61.8} & \multicolumn{2}{|c|}{$49 \cdot 6$} & \multicolumn{2}{|c|}{$36 \cdot 0$} \\
\hline European American (\%) & \multirow{2}{*}{\multicolumn{2}{|c|}{$\begin{array}{l}67 \cdot 7 \\
90 \cdot 3\end{array}$}} & \multirow{2}{*}{\multicolumn{2}{|c|}{$\begin{array}{l}72 \cdot 5 \\
84.3\end{array}$}} & \multirow{2}{*}{\multicolumn{2}{|c|}{$\begin{array}{l}68.8 \\
80.5\end{array}$}} & \multirow{2}{*}{\multicolumn{2}{|c|}{$\begin{array}{l}61.8 \\
88.2\end{array}$}} & \multirow{2}{*}{\multicolumn{2}{|c|}{$\begin{array}{l}59.3 \\
88.5\end{array}$}} & \multirow{2}{*}{\multicolumn{2}{|c|}{$\begin{array}{l}72.0 \\
74.0\end{array}$}} \\
\hline College degree (\%) & & & & & & & & & & & & \\
\hline Age (years) & $27 \cdot 4$ & 3.7 & $27 \cdot 0$ & 3.4 & $27 \cdot 0$ & 3.6 & 29.1 & 3.4 & 28.5 & 3.8 & $27 \cdot 4$ & 4.1 \\
\hline Height $(\mathrm{cm})$ & $168 \cdot 1$ & 9.4 & 171.3 & $10 \cdot 0$ & 171.9 & 8.4 & $169 \cdot 1$ & 8.6 & $171 \cdot 1$ & $9 \cdot 7$ & 171.8 & 9.2 \\
\hline Weight $(\mathrm{kg})$ & 64.7 & 8.6 & $66 \cdot 3$ & $9 \cdot 7$ & $66 \cdot 9$ & 8.9 & $85 \cdot 7$ & 8.0 & $84 \cdot 2$ & $12 \cdot 3$ & 83.5 & $12 \cdot 2$ \\
\hline BMI $\left(\mathrm{kg} / \mathrm{m}^{2}\right)$ & $22 \cdot 8$ & 1.5 & 22.5 & 1.6 & 22.5 & 1.5 & $30 \cdot 8$ & 3.1 & $28 \cdot \overline{7}$ & $2 \cdot 7$ & 28.2 & $2 \cdot 8$ \\
\hline Reported El $(\mathrm{kJ} / \mathrm{d})$ & 7973 & 2053 & 8869 & 2918 & 9049 & 2959 & 8511 & 2583 & 8506 & 2773 & 9076 & 2976 \\
\hline Reported El $(\mathrm{kcal} / \mathrm{d})$ & 1905.5 & $490 \cdot 6$ & $2119 \cdot 7$ & 697.3 & 2162.8 & $707 \cdot 3$ & 2034.1 & $617 \cdot 2$ & 2033.1 & $662 \cdot 8$ & $2169 \cdot 2$ & 711.3 \\
\hline TDEE $(\mathrm{kJ} / \mathrm{d})$ & 9397 & 1422 & 10606 & 1810 & 12071 & 2211 & 10863 & 1405 & 11715 & 1856 & 13178 & 2109 \\
\hline TDEE $(\mathrm{kcal} / \mathrm{d})$ & $2245 \cdot 9$ & 339.9 & 2534.8 & 432.5 & $2885 \cdot 1$ & 528.5 & $2596 \cdot 3$ & $335 \cdot 8$ & $2800 \cdot 0$ & 443.6 & 3149.7 & $504 \cdot 1$ \\
\hline MVPA $(\mathrm{min} / \mathrm{d})$ & 53.5 & 28.5 & 83.2 & 44.4 & 149.2 & $74 \cdot 3$ & $26 \cdot 3$ & 23.4 & 42.4 & $32 \cdot 2$ & 86.2 & 68.4 \\
\hline
\end{tabular}

$\mathrm{El}$, energy intake; TDEE, total daily energy expenditure; MVPA, moderate and vigorous physical activity (in bouts of at least 10 min with a minimum of 8 min at an intensity > 3 MET); MET, metabolic equivalent of task; PAL, physical activity level.

Values are percentages for categorical variables, means and standard deviations for continuous variables.

Sedentary $=\mathrm{PAL}<1.6$; active $=1.6 \leq \mathrm{PAL}<1.9$; vigorously active $=\mathrm{PAL} \geq 1.9$. 
Table 2 Macro- and micronutrient intakes of the sample of healthy young adult participants from the Energy Balance Study, by activity level in each weight category

\begin{tabular}{|c|c|c|c|c|c|c|c|c|c|c|c|c|}
\hline & \multicolumn{6}{|c|}{ Normal-weight group (n 210) } & \multicolumn{6}{|c|}{ Overweight/obese group ( $n$ 197) } \\
\hline & \multicolumn{2}{|c|}{$\begin{array}{l}\text { Sedentary } \\
\quad(n 31)\end{array}$} & \multicolumn{2}{|c|}{$\begin{array}{l}\text { Active } \\
(n \text { 102) }\end{array}$} & \multicolumn{2}{|c|}{$\begin{array}{l}\text { Vigorously active } \\
\qquad(n 77)\end{array}$} & \multicolumn{2}{|c|}{$\begin{array}{l}\text { Sedentary } \\
\quad(n 34)\end{array}$} & \multicolumn{2}{|c|}{$\begin{array}{l}\text { Active } \\
(n \text { 113) }\end{array}$} & \multicolumn{2}{|c|}{$\begin{array}{c}\text { Vigorously active } \\
(n 50)\end{array}$} \\
\hline & Mean & SD & Mean & SD & Mean & SD & Mean & SD & Mean & SD & Mean & SD \\
\hline \multicolumn{13}{|l|}{ Macronutrients } \\
\hline Protein (\% of El) & $15 \cdot 1$ & $3 \cdot 2$ & $16 \cdot 7$ & 3.9 & $16 \cdot 3$ & 3.9 & $17 \cdot 2$ & $4 \cdot 1$ & $18 \cdot 0$ & 5.9 & $19 \cdot 0$ & $5 \cdot 7$ \\
\hline Carbohydrate (\% of EI) & $50 \cdot 2$ & $9 \cdot 2$ & $47 \cdot 0$ & 8.7 & $49 \cdot 1$ & 9.8 & 46.9 & 9.5 & $46 \cdot 2$ & $10 \cdot 1$ & 44.8 & $10 \cdot 7$ \\
\hline Total fat (\% of El) & 31.9 & 7.5 & $32 \cdot 9$ & $7 \cdot 0$ & 31.8 & 7.9 & $33 \cdot 1$ & $7 \cdot 0$ & 33.5 & $7 \cdot 8$ & 33.3 & 6.5 \\
\hline Cholesterol (mg/d) & $232 \cdot 4$ & $154 \cdot 3$ & $284 \cdot 3$ & $190 \cdot 4$ & 255.0 & $170 \cdot 9$ & $296 \cdot 6$ & $214 \cdot 1$ & 298.0 & $206 \cdot 6$ & 358.9 & 429.5 \\
\hline Total fibre $(\mathrm{g} / \mathrm{d})^{\star}$ & 19.9 & 7.0 & $19 \cdot 6$ & 9.9 & 21.9 & 11.4 & 17.4 & $7 \cdot 3$ & $17 \cdot 7$ & $8 \cdot 1$ & 19.5 & $8 \cdot 1$ \\
\hline \multicolumn{13}{|l|}{ Minerals } \\
\hline $\mathrm{Ca}(\mathrm{mg} / \mathrm{d})$ & $900 \cdot 7$ & 353.1 & $949 \cdot 3$ & 491.9 & 944.5 & 393.4 & 918.8 & 475.9 & 924.6 & 623.6 & 998.2 & $446 \cdot 0$ \\
\hline $\mathrm{Fe}(\mathrm{mg} / \mathrm{d})$ & $16 \cdot 0$ & $6 \cdot 4$ & $15 \cdot 5$ & $6 \cdot 1$ & $16 \cdot 7$ & $6 \cdot 2$ & $15 \cdot 7$ & 5.9 & $15 \cdot 0$ & $5 \cdot 8$ & $16 \cdot 1$ & 6.5 \\
\hline$M g(m g / d) \dagger$ & $287 \cdot 7$ & $80 \cdot 5$ & $317 \cdot 7$ & $144 \cdot 1$ & $342 \cdot 8$ & $143 \cdot 1$ & $297 \cdot 7$ & 135.5 & $295 \cdot 1$ & $130 \cdot 3$ & $319 \cdot 8$ & $120 \cdot 7$ \\
\hline$P(\mathrm{mg} / \mathrm{d}) \dagger$ & $1180 \cdot 9$ & 314.4 & $1320 \cdot 5$ & $540 \cdot 8$ & $1338 \cdot 1$ & $438 \cdot 6$ & $1334 \cdot 2$ & 553.8 & $1310 \cdot 3$ & 645.7 & $1425 \cdot 4$ & 587.0 \\
\hline $\mathrm{K}(\mathrm{mg} / \mathrm{d})^{\star}, \dagger$ & $2484 \cdot 8$ & 786.7 & $2605 \cdot 8$ & $1200 \cdot 2$ & $2692 \cdot 3$ & $1084 \cdot 1$ & $2450 \cdot 3$ & $1018 \cdot 3$ & $2446 \cdot 7$ & $1046 \cdot 5$ & $2686 \cdot 2$ & $1003 \cdot 0$ \\
\hline $\mathrm{Na}(\mathrm{mg} / \mathrm{d})^{\star}, \dagger$ & $3186 \cdot 7$ & 882.5 & $3520 \cdot 1$ & 1283.9 & 3535.9 & $1207 \cdot 3$ & $3524 \cdot 7$ & 1201.9 & 3745.5 & 1293.5 & $3937 \cdot 1$ & $1418 \cdot 0$ \\
\hline $\mathrm{Zn}(\mathrm{mg} / \mathrm{d})$ & $11 \cdot 2$ & $6 \cdot 7$ & 11.7 & 5.4 & 11.6 & 4.6 & 11.4 & $6 \cdot 1$ & 11.5 & $5 \cdot 7$ & $12 \cdot 7$ & $6 \cdot 8$ \\
\hline $\mathrm{Cu}(\mathrm{mg} / \mathrm{d})^{\star}, \dagger$ & 1.3 & 0.4 & 1.4 & $0 \cdot 6$ & 1.6 & 0.7 & 1.3 & $0 \cdot 6$ & 1.3 & 0.5 & 1.4 & 0.5 \\
\hline $\operatorname{Se}(\mu \mathrm{g} / \mathrm{d}) \dagger$ & $98 \cdot 4$ & $27 \cdot 7$ & $118 \cdot 2$ & 44.9 & $125 \cdot 8$ & $55 \cdot 4$ & $120 \cdot 8$ & $47 \cdot 1$ & $117 \cdot 8$ & $45 \cdot 4$ & $135 \cdot 2$ & $67 \cdot 6$ \\
\hline \multicolumn{13}{|l|}{ Vitamins } \\
\hline Vitamin A ( $\mu \mathrm{g}$ RAE) & 2278.2 & $2196 \cdot 8$ & $2661 \cdot 7$ & 4043.6 & $2649 \cdot 4$ & 3129.5 & $2260 \cdot 9$ & $2960 \cdot 2$ & 2276.6 & $1756 \cdot 8$ & 2714.4 & $2501 \cdot 3$ \\
\hline Vitamin D $(\mu \mathrm{g} / \mathrm{d})$ & $3 \cdot 7$ & 1.9 & 4.0 & $3 \cdot 3$ & 3.9 & 2.5 & 4.3 & $3 \cdot 1$ & $4 \cdot 1$ & 3.8 & $5 \cdot 3$ & $7 \cdot 2$ \\
\hline Vitamin $E(\mathrm{mg} / \mathrm{d}) \ddagger$ & $17 \cdot 5$ & $19 \cdot 7$ & $14 \cdot 2$ & $10 \cdot 7$ & $15 \cdot 8$ & $12 \cdot 9$ & $11 \cdot 8$ & $7 \cdot 8$ & $13 \cdot 9$ & 9.9 & 14.4 & 9.9 \\
\hline Vitamin $C(\mathrm{mg} / \mathrm{d})$ & $90 \cdot 6$ & 55.5 & $90 \cdot 6$ & 94.7 & 93.9 & 71.4 & 78.9 & 53.3 & $85 \cdot 7$ & 58.5 & $75 \cdot 7$ & $46 \cdot 6$ \\
\hline Thiamin $(\mathrm{mg} / \mathrm{d}) \dagger$ & 1.7 & 0.7 & 1.7 & 0.7 & 1.9 & 0.7 & 1.8 & 0.6 & 1.7 & 0.6 & 1.8 & 0.7 \\
\hline Riboflavin $(\mathrm{mg} / \mathrm{d}) \dagger$ & $2 \cdot 0$ & 0.7 & $2 \cdot 2$ & 1.0 & $2 \cdot 3$ & 0.9 & $2 \cdot 3$ & 1.0 & $2 \cdot 0$ & 1.2 & 2.5 & 1.1 \\
\hline Niacin $(\mathrm{mg} / \mathrm{d}) \dagger$ & 23.9 & $8 \cdot 3$ & 26.5 & $11 \cdot 6$ & $28 \cdot 8$ & 11.6 & $26 \cdot 1$ & $11 \cdot 7$ & $26 \cdot 3$ & 11.9 & $30 \cdot 2$ & 11.9 \\
\hline Folate $(\mu \mathrm{g} / \mathrm{d})$ & $456 \cdot 3$ & 192.9 & $460 \cdot 8$ & $190 \cdot 1$ & 502.9 & $222 \cdot 0$ & $427 \cdot 7$ & $165 \cdot 6$ & $442 \cdot 4$ & $205 \cdot 7$ & $457 \cdot 7$ & $194 \cdot 3$ \\
\hline Vitamin $B_{6}(\mathrm{mg} / \mathrm{d}) \dagger$ & $2 \cdot 1$ & 1.0 & $2 \cdot 2$ & $1 \cdot 1$ & $2 \cdot 6$ & 1.6 & $2 \cdot 1$ & $1 \cdot 1$ & 2.5 & $3 \cdot 5$ & $2 \cdot 4$ & $1 \cdot 2$ \\
\hline Vitamin $B_{12}(\mu \mathrm{g} / \mathrm{d}) \dagger$ & 4.0 & $2 \cdot 0$ & 4.7 & $2 \cdot 7$ & $5 \cdot 1$ & 3.4 & $5 \cdot 0$ & 3.0 & $6 \cdot 1$ & $15 \cdot 6$ & $5 \cdot 7$ & $5 \cdot 0$ \\
\hline Choline $(\mathrm{mg} / \mathrm{d}) \dagger$ & $280 \cdot 2$ & $116 \cdot 1$ & $338 \cdot 3$ & $166 \cdot 5$ & $327 \cdot 2$ & $137 \cdot 1$ & $338 \cdot 0$ & $192 \cdot 0$ & $340 \cdot 2$ & $175 \cdot 0$ & $398 \cdot 0$ & $316 \cdot 3$ \\
\hline Vitamin $\mathrm{K}(\mu \mathrm{g} / \mathrm{d})$ & $105 \cdot 5$ & $67 \cdot 8$ & 197.5 & 581.9 & 134.54 & $108 \cdot 8$ & 131.9 & $101 \cdot 8$ & 151.5 & 124.9 & $141 \cdot 1$ & $198 \cdot 2$ \\
\hline
\end{tabular}

El, energy intake; RAE, retinol activity equivalents (RAE vitamin $A=\mu \mathrm{g}$ retinol $+(\mu \mathrm{g} \beta$-carotene equivalents $/ 12)$ ).

Sedentary $=\mathrm{PAL}<1.6$; active $=1.6 \leq \mathrm{PAL}<1.9$; vigorously active $=\mathrm{PAL} \geq 1.9$.

${ }^{*} P$ for trend $<0.05$ in overweight/obese adults after adjusting for age, sex, social desirability, social approval and El difference.

$\dagger P$ for trend $<0.05$ in normal-weight adults adjusting for age, sex, social desirability, social approval and El difference.

$\ddagger P$ for trend $<0.05$ in normal-weight adults after additionally adjusting for reported $\mathrm{El}$.
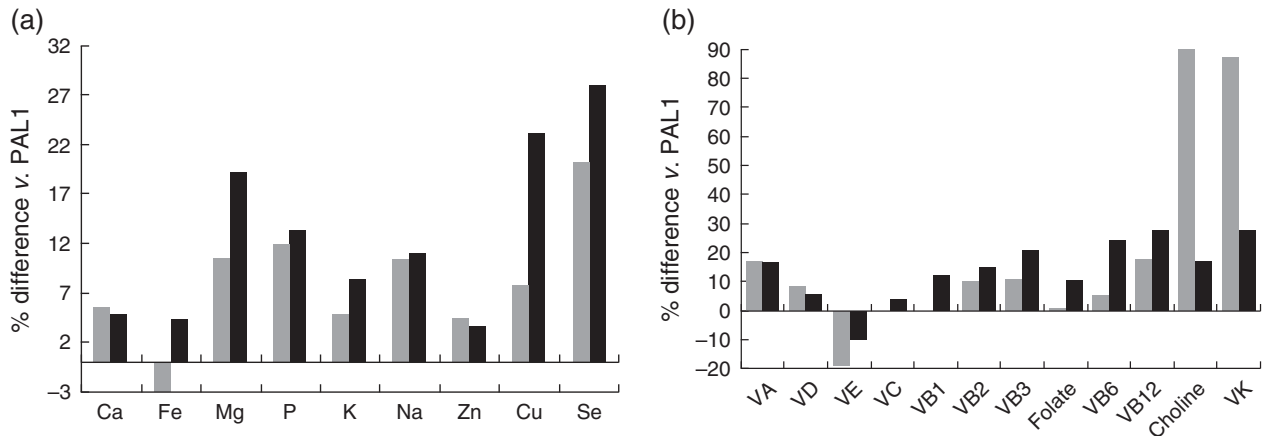

Fig. 1 Percentage difference in the intakes of (a) minerals and (b) vitamins in active (PAL2; $\square$ ) and vigorously active (PAL3; $\square$ ) participants compared with sedentary (PAL1) participants in the normal-weight group adjusting for age, sex, social desirability, social approval and El difference; sample of healthy young adult participants from the Energy Balance Study. The mineral or vitamin \% difference of PAL2 $v$. PAL1 is equal to [(reported mineral or vitamin intake PAL2 $_{-}$reported mineral or vitamin intake PAL1 $\left._{1}\right) /$ reported mineral or vitamin intake PAL1 $] \times 100 \%$; the mineral or vitamin $\%$ difference of PAL3 $v$. PAL1 is equal to [(reported mineral or vitamin intake $e_{\text {PAL3 }}$ - reported mineral or vitamin intake $\left.e_{\text {PAL1 }}\right) /$ reported mineral or vitamin intake $\left.e_{\text {PAL1 }}\right) \times 100 \%$ (PAL, physical activity (sedentary $=\mathrm{PAL}<1.6$, active $=1.6 \leq \mathrm{PAL}<1.9$, vigorously active $=\mathrm{PAL} \geq 1.9$ ); $\mathrm{EI}$, energy intake; $\mathrm{VA}$, vitamin A; VD, vitamin D; VE, vitamin E; VC, vitamin C; VB1, thiamin; VB2, riboflavin; VB3, niacin; VB6, vitamin B $_{6}$; VB12, vitamin $\mathrm{B}_{12}$, VK, vitamin $\left.\mathrm{K}\right)$ 


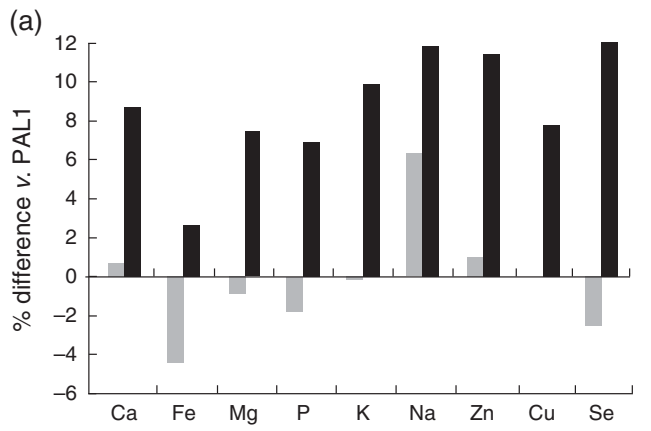

(b)

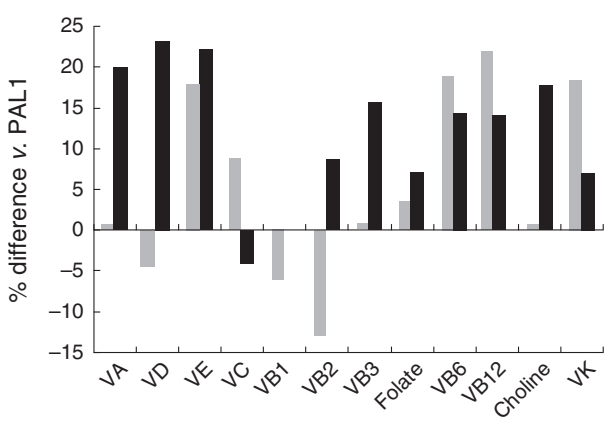

Fig. 2 Percentage difference in the intakes of (a) minerals and (b) vitamins in active (PAL2; $\square$ ) and vigorously active (PAL3; $\square$ ) participants compared with sedentary (PAL1) participants in the overweight/obese group adjusting for age, sex, social desirability, social approval and El difference; sample of healthy young adult participants from the Energy Balance Study. The mineral or vitamin $\%$ difference of PAL2 $v$. PAL1 is equal to [(reported mineral or vitamin intake PAL2 $_{-}$reported mineral or vitamin intake $\left.e_{\text {PAL1 }}\right) /$ reported mineral or vitamin intake $\left.\mathrm{PAL}_{1}\right] \times 100 \%$; the mineral or vitamin $\%$ difference of PAL3 $v$. PAL1 is equal to [(reported mineral or vitamin intake $e_{\mathrm{PAL} 3}$ - reported mineral or vitamin intake $\left.\mathrm{PAL1}_{1}\right) /$ reported mineral or vitamin intake $\left.\mathrm{PAL}_{1}\right) \times 100 \%$ (PAL, physical activity (sedentary $=P A L<1.6$, active $=1.6 \leq P A L<1.9$, vigorously active $=P A L \geq 1.9$ ); El, energy intake; VA, vitamin $A$; VD, vitamin $D$; VE, vitamin E; VC, vitamin C; VB1, thiamin; VB2, riboflavin; VB3, niacin; VB6, vitamin $\mathrm{B}_{6}$; VB12, vitamin $\mathrm{B}_{12}$, VK, vitamin K)

intakes no longer differed when total energy intake was taken into consideration. PA per se, therefore, may not affect dietary pattern. However, higher energy expenditure due to increased PA would allow for greater food consumption while maintaining energy balance. Individuals who want to maintain body weight at low levels of energy expenditure must sustain food restriction over the long term, which may not be feasible for most people. Hence, at low levels of energy expenditure individuals may continually be at risk of gaining weight or insufficient nutrient intake. It has further been hypothesized that there is a minimum threshold for energy expenditure above which energy intake is more easily matched to expenditure through appetite control ${ }^{(37)}$. Results of the present study suggest that PA does not necessarily affect dietary pattern but it does allow for a higher energy intake, which could contribute to dietary nutrient adequacy and provide health benefits in excess of supplements. This is an additional argument for the importance of PA regarding overall health.

Consistent with various observations in other Western populations ${ }^{(2,7,38,39)}$, our results indicated that sedentary young adults consumed lower amounts of essential minerals and vitamins from the array of foods while meeting their energy needs. Diets low in energy and nutrients, to compensate for increasingly sedentary lifestyles, may, however, have detrimental effects on overall health. Even though these individuals try to reduce chronic disease risk with weight maintenance, they may have nutrient inadequacies that increase their risk for some chronic diseases ${ }^{(40,41)}$. For example, dietary fibre is good for promoting intestinal peristalsis and preventing colon cancer. It has been further shown that healthyweight participants with a higher PAL consume more dietary fibre, which is associated with a lesser degree of weight gain ${ }^{(42)}$. Consumption of an additional $14 \mathrm{~g}$ fibre/d for $>2 \mathrm{~d}$ is associated with a $10 \%$ decrease in energy intake due to an increase in post-meal satiety and a decrease in subsequent hunger ${ }^{(8)}$. The present study showed that higher PAL is associated with higher fibre intake, particularly in normal-weight participants.

Minerals and vitamins are key nutrients for health maintenance and chronic disease prevention ${ }^{(43)}$. For example, $\mathrm{Ca}, \mathrm{Mg}$ and $\mathrm{K}$ are crucial in building and maintaining bone mineral density ${ }^{(44)}$. Dietary $\mathrm{Ca}, \mathrm{Mg}$ and $\mathrm{K}$ combined with plant sterols have been suggested as a promising novel approach to modifying CVD risk ${ }^{(10,45)}$. Insufficient intakes of $\mathrm{Ca}, \mathrm{Mg}$ and $\mathrm{K}$ and high $\mathrm{Na}$ intake are associated with arterial hypertension ${ }^{(7,9)}$. The B-group vitamins are essential for energy production. Such as, thiamin helps to convert glucose into energy and has a role in nerve function; riboflavin is primarily involved in energy production and helps vision and skin health; niacin is essential for the body to convert carbohydrates, fat and alcohol into energy, helps maintain skin health and supports the nervous and digestive systems. As was addressed for fibre intake, higher PAL was associated with higher intakes of $\mathrm{Mg}$ and $\mathrm{K}$ in normal-weight, but not in overweight/obese participants. Although increasing energy expenditure and energy intake alone may not be sufficient to correct the dietary inadequacies, the present results indicate that higher PA is associated with higher intakes of vitamins and minerals, which are associated with various health benefits.

Several limitations need to be considered when interpreting the findings of the present study. The sample consisted predominantly of European-American, well-educated adults with a high activity level (average PAL $=1 \cdot 7$ ), which limits the generalizability of the study results. Further, dietary intake was assessed via self-report, which has inherent 
sources of measurement error. In order to increase the accuracy of dietary reports, participants were given a validated two-dimensional food portion visual and received 10-15 min of training on how to use it to estimate portion sizes of commonly eaten foods ${ }^{(46)}$. To help account for dietary misreporting, social desirability and social approval were also considered in the analysis as these have been shown to bias dietary assessment ${ }^{(30-33)}$. In addition, calculated energy requirements were included as a covariate in order to adjust for misreporting of overall dietary intake. It also should also be considered that dietary supplements were not included in the assessment of dietary intake in the present study. Although most people do not consume an optimal amount of all vitamins by diet alone, at least $50 \%$ of adults in the USA take dietary supplements ${ }^{(47,48)}$; evidence on the relationship between dietary supplements and health risk is still inconsistent ${ }^{(49,50)}$ and adequate nutrient intake via regular foods remains a safer source of nutrients.

\section{Conclusion}

In summary, the present study showed that individuals with a higher PAL have higher intakes of key nutrients. Dietary pattern, however, does not seem to differ significantly between PA categories, suggesting that the benefits of PA are predominantly due to a higher overall food intake while maintaining energy balance, rather than a healthier diet.

\section{Acknowledgements}

Acknowledgements: The authors wish to thank the study participants and the Energy Balance Study team. Financial support: Funding for this project was provided through a grant from The Coca-Cola Company. The sponsor played no role in the study design, collection, analysis and interpretation of data, or preparation and submission of this manuscript. Authorship: G.A.H., S.N.B. and J.R.H. conceived and designed the study. R.P.S. managed data collection. Y.Y. analysed the data and wrote the manuscript with input from C.D., J.R.H., R.P.S., T.G.H., G.A.H. and S.N.B. Etbics of human subject participation: The study was approved by the University of South Carolina Institutional Review Board and all participants signed informed consent prior to data collection.

\section{References}

1. Allison DB, Fontaine KR, Manson JE et al. (1999) Annual deaths attributable to obesity in the United States. JAMA 282, 1530-1538.

2. Csizmadi I, Kelemen LE, Speidel T et al. (2014) Are physical activity levels linked to nutrient adequacy? Implications for cancer risk. Nutr Cancer 66, 214-224.

3. Haslam DW \& James WPT (2005) Obesity. Lancet $\mathbf{3 6 6}$, 1197-1209.
4. Esposito K, Kastorini CM, Panagiotakos DB et al. (2011) Mediterranean diet and weight loss: meta-analysis of randomized controlled trials. Metab Syndr Relat Disord 9 , $1-12$.

5. US Department of Agriculture \& US Department of Health and Human Services (2010) Dietary Guidelines for Americans, 2010, 7th ed. Washington, DC: US Government Printing office; available at http://www.cnpp.usda.gov/ DietaryGuidelines

6. Caballero B (2007) The global epidemic of obesity: an overview. Epidemiol Rev 29, 1-5.

7. Karppanen H, Karppanen P \& Mervaala E (2005) Why and how to implement sodium, potassium, calcium, and magnesium changes in food items and diets? J Hum Hypertens 19, Suppl. 3, S10-S19.

8. Howarth NC, Saltzman E \& Roberts SB (2001) Dietary fiber and weight regulation. Nutr Rev 59, 129-139.

9. Karppanen H (1991) Minerals and blood pressure. Ann Med 23, 299-305.

10. Vaskonen T (2003) Dietary minerals and modification of cardiovascular risk factors. $J$ Nutr Biochem 14, 492-506.

11. Garriguet D (2011) Bone health: osteoporosis, calcium and vitamin D. Health Rep 22, 7-14.

12. Cao ZB, Sasaki A, Oh T et al. (2012) Association between dietary intake of micronutrients and cardiorespiratory fitness in Japanese men. J Nutr Sci 1, e12.

13. Carlson JA, Sallis JF, Ramirez ER et al. (2012) Physical activity and dietary behavior change in Internet-based weight loss interventions: comparing two multiplebehavior change indices. Prev Med 54, 50-54.

14. Westerterp KR (2010) Physical activity, food intake, and body weight regulation: insights from doubly labeled water studies. Nutr Rev 68, 148-154.

15. Shay CM, Van Horn L, Stamler J et al. (2012) Food and nutrient intakes and their associations with lower BMI in middle-aged US adults: the International Study of Macro-/ Micronutrients and Blood Pressure (INTERMAP). Am J Clin Nutr 96, 483-491.

16. Kaur B \& Henry J (2014) Micronutrient status in type 2 diabetes: a review. Adv Food Nutr Res 71, 55-100.

17. Zemel MB, Donnelly JE, Smith BK et al. (2008) Effects of dairy intake on weight maintenance. Nutr Metab (Lond) 5, 28.

18. Proper KI, Singh AS, van Mechelen W et al. (2011) Sedentary behaviors and health outcomes among adults: a systematic review of prospective studies. Am J Prev Med 40, 174-182.

19. Hand GA, Shook RP, Paluch AE et al. (2013) The energy balance study: the design and baseline results for a longitudinal study of energy balance. Res Q Exerc Sport 84, $275-286$.

20. Lang A \& Froelicher ES (2006) Management of overweight and obesity in adults: behavioral intervention for long-term weight loss and maintenance. Eur J Cardiovasc Nurs 5, 102-114.

21. St-Onge M, Mignault D, Allison DB et al. (2007) Evaluation of a portable device to measure daily energy expenditure in free-living adults. Am J Clin Nutr 85, 742-749.

22. Welk GJ, McClain JJ, Eisenmann JC et al. (2007) Field validation of the MTI Actigraph and BodyMedia armband monitor using the IDEEA monitor. Obesity (Silver Spring) 15, 918-928.

23. Ainsworth BE, Haskell WL, Herrmann SD et al. (2011) 2011 Compendium of Physical Activities: a second update of codes and MET values. Med Sci Sports Exerc 43, 1575-1581.

24. Food and Nutrition Board, Institute of Medicine (2002) Dietary Reference Intakes for Energy, Carbohydrate, Fiber, Fat, Fatty Acids, Cholesterol, Protein, and Amino Acids (Macronutrients). Washington, DC: The National Academies Press. 
25. Novotny JA, Rumpler WV, Judd JT et al. (2001) Diet interviews of subject pairs: how different persons recall eating the same foods. J Am Diet Assoc 101, 1189-1193.

26. Ma Y, Olendzki BC, Pagoto SL et al. (2009) Number of 24-hour diet recalls needed to estimate energy intake. Ann Epidemiol 19, 553-559.

27. Thomas DM, Schoeller DA, Redman LA et al. (2010) A computational model to determine energy intake during weight loss. Am J Clin Nutr 92, 1326-1331.

28. Crowne DP \& Marlowe D (1960) A new scale of social desirability independent of psychopathology. J Consult Psychol 24, 349-354.

29. Martin HJ (1984) A revised measure of approval motivation and its relationship to social desirability. J Pers Assess 48, 508-519.

30. Hebert JR, Hurley TG, Peterson KE et al. (2008) Social desirability trait influences on self-reported dietary measures among diverse participants in a multicenter multiple risk factor trial. $J$ Nutr 138, issue 1, 226S-234S.

31. Hebert JR, Peterson KE, Hurley TG et al. (2001) The effect of social desirability trait on self-reported dietary measures among multi-ethnic female health center employees. Ann Epidemiol 11, 417-427.

32. Hebert JR, Clemow L, Pbert L et al. (1995) Social desirability bias in dietary self-report may compromise the validity of dietary intake measures. Int J Epidemiol 24, 389-398.

33. Hebert JR, Ma Y, Clemow L et al. (1997) Gender differences in social desirability and social approval bias in dietary self-report. Am J Epidemiol 146, 1046-1055.

34. Fogelholm M, Anderssen S, Gunnarsdottir I et al. (2012) Dietary macronutrients and food consumption as determinants of long-term weight change in adult populations: a systematic literature review. Food Nutr Res 2012, 56.

35. Parsons TJ, Power C \& Manor O (2006) Longitudinal physical activity and diet patterns in the 1958 British Birth Cohort. Med Sci Sports Exerc 38, 547-554.

36. Brodney S, McPherson RS, Carpenter RS et al. (2001) Nutrient intake of physically fit and unfit men and women. Med Sci Sports Exerc 33, 459-467.

37. Hill JO, Wyatt HR \& Peters JC (2012) Energy balance and obesity. Circulation 126, 126-132.
38. Anderson LN, Cotterchio M, Boucher BA et al. (2010) Vitamin D intake from food and supplements among Ontario women based on the US block food frequency questionnaire with and without modification for Canadian food values. Can J Public Health 101, 318-321.

39. Garriguet D (2010) The effect of supplement use on vitamin C intake. Health Rep 21, 57-62.

40. Fairfield KM \& Fletcher RH (2002) Vitamins for chronic disease prevention in adults: scientific review. JAMA $\mathbf{2 8 7}$, 3116-3126.

41. Fletcher RH \& Fairfield KM (2002) Vitamins for chronic disease prevention in adults: clinical applications. JAMA 287, 3127-3129.

42. van Dam RM \& Seidell JC (2007) Carbohydrate intake and obesity. Eur J Clin Nutr 61, Suppl. 1, S75-S99.

43. Ramage-Morin PL \& Garriguet D (2013) Nutritional risk among older Canadians. Health Rep 24, 3-13.

44. Sarkis KS, Pinheiro Mde M, Szejnfeld VL et al. (2012) High bone density and bone health. Endocrinol Nutr 59, 207-214.

45. Vaskonen T, Mervaala E, Krogerus L et al. (2002) Supplementation of plant sterols and minerals benefits obese Zucker rats fed an atherogenic diet. J Nutr 132, 231-237.

46. Posner BM, Smigelski C, Duggal A et al. (1992) Validation of two-dimensional models for estimation of portion size in nutrition research. J Am Diet Assoc 92, 738-741.

47. Millen AE, Dodd KW \& Subar AF (2004) Use of vitamin, mineral, nonvitamin, and nonmineral supplements in the United States: the 1987, 1992, and 2000 National Health Interview Survey results. J Am Diet Assoc 104, 942-950.

48. Radimer K, Bindewald B, Hughes J et al. (2004) Dietary supplement use by US adults: data from the National Health and Nutrition Examination Survey, 1999-2000. Am J Epidemiol 160, 339-349.

49. Park SY, Murphy SP, Wilkens LR et al. (2011) Multivitamin use and the risk of mortality and cancer incidence: the multiethnic cohort study. Am J Epidemiol 173, 906-914.

50. Pocobelli G, Peters U, Kristal AR et al. (2009) Use of supplements of multivitamins, vitamin $\mathrm{C}$, and vitamin $\mathrm{E}$ in relation to mortality. Am J Epidemiol 170, 472-483. 